\title{
Interplay and optimization of decoherence mechanisms in the optical control of spin quantum bits implemented on a semiconductor quantum dot
}

\author{
A. Grodecka,,${ }^{1,2, *}$ C. Weber,${ }^{1}$ P. Machnikowski, ${ }^{2}$ and A. Knorr ${ }^{1}$ \\ ${ }^{1}$ Insitut für Theoretische Physik, Nichtlineare Optik und Quantenelektronik, \\ Technische Universität Berlin, 10623 Berlin, Germany \\ ${ }^{2}$ Institute of Physics, Wroctaw University of Technology, 50-370 Wroctaw, Poland
}

\begin{abstract}
We study the influence of the environment on an optically induced rotation of a single electron spin in a charged semiconductor quantum dot. We analyze the decoherence mechanisms resulting from the dynamical lattice response to the charge evolution induced in a trion-based optical spin control scheme. Moreover, we study the effect of the finite trion lifetime and of the imperfections of the unitary evolution such as off-resonant excitations and the nonadiabaticity of the driving. We calculate the total error of the operation on a spin-based qubit in an InAs/GaAs quantum dot system and discuss possible optimization against the different contributions. We indicate the parameters which allow for coherent control of the spin with a single qubit gate error as low as $10^{-4}$.

PACS numbers: $63.20 . \mathrm{Kr}, 03.65 . \mathrm{Yz}, 03.67 . \mathrm{Lx}, 42.50 . \mathrm{Hz}, 71.35 . \mathrm{Pq}$
\end{abstract}

\section{INTRODUCTION}

The spin degree of freedom of an excess electron in a charged semiconductor quantum dot (QD) has been proposed as an attractive candidate for the use as a qubit $1,2,3$. The advantage of storing the logical values in spin states is their long coherence time ${ }^{4}$. In addition, it is possible to optically induce charge dynamics dependent on the spin state of an electron via the Pauli exclusion principle and optical selection rules 5.6 .7 . Coupling between charge states via statio $\frac{1.5}{1.5}$ and interband ${ }^{8.9}$ electric dipole moments allows one to perform quantum conditional operations on two spins. In this way, one can implement single- and two-qubit gates in QD systems. The utilization of the optical control methods leads to shorter switching times, on a picosecond time scale, in comparison with nanosecond magnetic control of the spin, which is essential for the implementation of quantum information processing schemes. The capability of encoding and manipulating information at the single-spin level is of great importance and has been experimentally demonstrated recently: the generation ${ }^{10}$ and optical control ${ }^{11,12}$ of the spin coherence together with a possible read-out of the state of a single confined spin in a QD system 13 can make the implementation of a quantum computer feasible.

A promising scheme of quantum optical control of a spin in a single QD was recently proposed in Ref. 14. It has been shown that coupling to a trion (charged exciton) state leads to an arbitrary rotation between the two Zeeman-split spin states. In this way, optical coherent control of a spin in a QD via adiabatic Raman transitions is possible. This control protocol does not require an auxiliary fourth state which was needed in a similar scheme previously proposed ${ }^{15}$, removing the requirement for the transfer of the electron between two QDs and the delocalized hole state.

In realistic experiments, the QDs used for the proposed implementation are embedded in a solid state matrix, and confined carriers interact with the phonon bath, leading to a loss of coherence. In optical spin control schemes, spin rotation is achieved by spin-dependent charge evolution. Therefore, the spin decoherence in these schemes results mainly from the lattice response to the evolving charge density 1 16,17,18 . This effect has been studied ${ }^{17}$ for a control scheme using an auxiliary state $\frac{15}{}$ but not for the scheme of Ref. 14 that seems to be advantageous in some respects. Because of the different scheme of control the phonon-induced errors in the latter case have a different form and can be attributed to two channels: pure dephasing and phonon-assisted trion generation. Furthermore, since in this control procedure the spin rotation involves a finite trion occupation (unlike in the other scheme $\frac{15}{5}$ ), in addition to the phonon interaction the finite trion lifetime can also lead to decoherence, because of the nonzero probability of the radiative decay of the $\operatorname{trion}^{19}$. Moreover, the operation in the ideal case should be performed adiabatically and the imperfect adiabaticity of the evolution will contribute to the total error of the operation. Finally, since this control scheme involves spectral selection of transitions (in contrast to the other one), off-resonant terms can also lead to an unwanted leakage to the trion state and result in large discrepancies in the desired spin rotation. It is not clear in advance which of these factors (if any) will dominate the decoherence under specific driving conditions. So far, for the spin control scheme of Ref. 14, only the error resulting from imperfect adiabaticity of the driving and from the finite trion lifetime was evaluated ${ }^{19}$ but the impact of phonons, off-resonant excitations, and the joint decoherence effect have not been studied.

In this paper, we study the combined influence of the phonon and photon environments and of the imperfections of the evolution on an optical spin control scheme based on an off-resonant coupling of the spin states to a trion state in a doped semiconductor $\mathrm{QD}^{14}$. As a 
single-qubit gate, an optically induced arbitrary rotation of the electron spin state is considered. We show that the interactions with phonons and photons are the dominant sources of decoherence in this system. The phononinduced decoherence has two origins: pure dephasing and phonon-assisted trion generation, with a different dependence on the duration and the detuning of the optical control pulses. We show that by slowing down the evolution one can considerably decrease only the error due to the pure dephasing, while the phonon-assisted trion generation part still results in a large operation error. The contributions to the error resulting from the finite lifetime of the trion and the imperfections of the evolution are also studied. We calculate the total error of the operation and study the nontrivial interplay of the different contributions. In particular, we show that for moderate detunings $(\sim 1 \mathrm{meV})$ and long pulse durations the error is dominated by phonon-related effects. We indicate the optimal conditions for the qubit rotation for which the error is considerably small, with values even below $10^{-4}$, which is essential for coherent quantum control, and discuss the possible optimization against particular contributions to the error.

The paper is organized as follows. In Sec. II, we introduce the model for the qubit based on the spin states of a confined electron in a charged QD. Next, in Sec. III. we describe a single-qubit rotation scheme. In Sec. IV] we study the imperfections of the evolution. Section $\mathrm{V}$ describes the general perturbative method for describing the effects of the environment on an arbitrary operation on a logical qubit. Section VI contains the results for the error contributions due to the interaction of the carriers with the phonon and photon environments. In Sec. VII. we calculate the total error of the spin rotation and discuss the interplay and possible optimization of the different sources of the error. Section VIII concludes the paper with final remarks. In addition, some technical details are presented in the Appendixes.

\section{MODEL SYSTEM}

We consider a single semiconductor QD charged with one additional electron. A magnetic field applied in the $x$ direction [see Fig. 1(a)] causes a Zeeman splitting $\Delta_{\mathrm{B}}$ between the electron states with spin-up and spin-down (with respect to the direction of the magnetic field) which define the two logical qubit states $|0\rangle$ and $|1\rangle$ in the proposed scheme 14 . These two states are off-resonantly coupled (with a detuning $\Delta$ ) to a trion state $|2\rangle$ by $\sigma_{+}-$ polarized laser pulses with real amplitudes $\Omega_{0}(t)$ and $\Omega_{1}(t)$ and with different phases [see Fig. 1(a,b)]. These two electron spin states $|0\rangle$ and $|1\rangle$ together with the trion state $|2\rangle$ compose a three-level system, known in the literature as a $\Lambda$ system 20 .

The Hamiltonian of the system is given by

$$
H=H_{\mathrm{C}}+H_{\mathrm{env}}+V
$$

where the first term (control Hamiltonian) describes the carriers and their interaction with the classical driving field (the laser beam) and the second is the sum of the free phonon $H_{\mathrm{ph}}$ and photon $H_{\mathrm{rad}}$ contributions. The last part, $V=H_{\mathrm{c}-\mathrm{ph}}+H_{\mathrm{c}-\mathrm{rad}}$, describes the coupling of the carriers to the environment, where the first term denotes the carrier-phonon interaction and the second describes the coupling of the carriers to the photon field.

The control Hamiltonian for this system in dipole and rotating wave approximations is

$$
\begin{aligned}
H_{\mathrm{C}}= & \sum_{n} \epsilon_{n}|n\rangle\langle n| \\
& +\frac{\hbar}{2}\left[\sum_{n=0,1} \Omega_{n}(t) e^{i\left(\omega_{n} t+\gamma_{n}\right)}(|0\rangle+|1\rangle)\langle 2|+\text { H.c. }\right],
\end{aligned}
$$

where $\epsilon_{n}$ are the energies of the corresponding states, $\omega_{n}$ are the laser frequencies, and $\gamma_{n}$ are the phases of the pulses. The frequencies $\omega_{0}$ and $\omega_{1}$ have to satisfy the Raman conditions, namely that the detunings from the corresponding transition energies $\epsilon_{2}-\epsilon_{n}$ should be the same. To this end, we set $\omega_{n}=\left(\epsilon_{2}-\epsilon_{n}\right) / \hbar-\Delta$ $(n=0,1)$, where $\Delta$ is the common Raman detuning. We perform a unitary transformation to the rotating frame with $|\tilde{n}\rangle=e^{i\left(\omega_{n} t-\gamma_{0}\right)}|n\rangle(n=0,1)$. We can set $\gamma_{0}=\gamma$, $\gamma_{1}=0$ because only the relative phase is important. In the rotating frame, the Hamiltonian reads

$$
\begin{aligned}
H_{\mathrm{C}}= & \hbar \Delta|2\rangle\langle 2|+\frac{\hbar}{2} \Omega_{0}(t)\left(e^{i \gamma}|\tilde{0}\rangle\left\langle 2\left|+e^{-i \gamma}\right| 2\right\rangle\langle\tilde{0}|\right) \\
& +\frac{\hbar}{2} \Omega_{1}(t)(|\tilde{1}\rangle\langle 2|+| 2\rangle\langle\tilde{1}|)+H_{\mathrm{C}}^{\prime},
\end{aligned}
$$

where

$$
H_{\mathrm{C}}^{\prime}=\frac{\hbar}{2} \Omega_{1}(t) e^{i \Delta_{\mathrm{B}} t}|\tilde{0}\rangle\left\langle 2\left|+\frac{\hbar}{2} \Omega_{0}(t) e^{-i \Delta_{\mathrm{B}} t+i \gamma}\right| \tilde{1}\right\rangle\langle 2|+\text { H.c. }
$$

contains oscillating (off-resonant) terms which can be treated as a perturbation to the ideal evolution generated by the Hamiltonian given in Eq. (1).
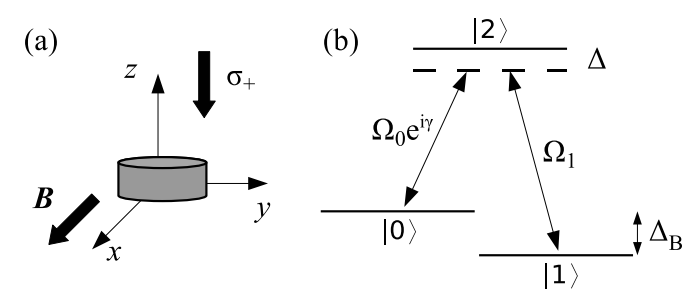

FIG. 1: (a) Schematic plot of the QD with the direction of incidence of the $\sigma_{+}$-polarized laser beam and the orientation of the magnetic field. (b) Three-level system in a single doped QD. 
The free phonon Hamiltonian has the form

$$
H_{\mathrm{ph}}=\sum_{\boldsymbol{k}} \hbar \omega_{\boldsymbol{k}} \beta_{\boldsymbol{k}}^{\dagger} \beta_{\boldsymbol{k}}
$$

where $\beta_{\boldsymbol{k}}^{\dagger}$ and $\beta_{\boldsymbol{k}}$ are phonon creation and annihilation operators, respectively, with corresponding frequencies $\omega_{\boldsymbol{k}}$, where $\boldsymbol{k}$ is the phonon wave number. The unperturbed photon Hamiltonian in the absence of charges reads

$$
H_{\mathrm{rad}}=\sum_{\mathbf{q}, \lambda} \hbar \omega_{\mathbf{q}}^{\prime} c_{\mathbf{q} \lambda}^{\dagger} c_{\mathbf{q} \lambda}
$$

with photon creation and annihilation operators $c_{\mathbf{q} \lambda}^{\dagger}$ and $c_{\mathbf{q} \lambda}$, respectively. Here, $\omega_{\mathbf{q}}^{\prime}=c|\mathbf{q}| / n_{\mathrm{r}}$ is the photon frequency, with the speed of light in vacuum $c$, the photon wave number $\mathbf{q}$, and the refractive index of the semiconductor medium $n_{\mathrm{r}} ; \lambda$ labels the polarization. The unperturbed evolution of the system and of the decoupled environment is described by $H_{\mathrm{C}}+H_{\mathrm{ph}}+H_{\mathrm{rad}}$.

The interaction of the carriers with the environment includes the coupling to phonons and photons. The carrierphonon interaction reads

$$
H_{\mathrm{c}-\mathrm{ph}}=\sum_{n, n^{\prime}}|n\rangle\left\langle n^{\prime}\right| \sum_{\boldsymbol{k}} f_{n n^{\prime}}(\boldsymbol{k})\left(\beta_{\boldsymbol{k}}+\beta_{-\boldsymbol{k}}^{\dagger}\right)
$$

with coupling constants $f_{n n^{\prime}}(\boldsymbol{k})$ having the symmetry $f_{n n^{\prime}}(\boldsymbol{k})=f_{n^{\prime} n}^{*}(-\boldsymbol{k})$. The states $|0\rangle$ and $|1\rangle$ correspond to a single electron confined in the same QD structure and differ only by the spin orientation. Therefore, they have the same orbital wave functions, and thus the coupling constants $f_{00}(\boldsymbol{k})$ and $f_{11}(\boldsymbol{k})$ are the same. These states have different spins so that $f_{01}(\boldsymbol{k})$ and $f_{10}(\boldsymbol{k})$ would describe a 'direct' phonon-assisted spin flip, mediated by the spin-orbit coupling 21 . However, in the optical spincontrol schemes, the effect of this process is many orders of magnitude weaker than the decoherence due to the dynamical response to charge evolution $\frac{17}{}$. Therefore, we neglect this coupling and set these coefficients equal to zero.

Initially, before the arrival of the pulses, the lattice is in a configuration where one electron is present in the QD surrounded by a lattice deformation (a polaronlike $\operatorname{state}^{22,23,24}$ ). We redefine the phonon modes to obtain the ground state of the carrier-phonon system corresponding to this new lattice equilibrium. In terms of the new phonon operators

$$
b_{\boldsymbol{k}}=\beta_{\boldsymbol{k}}+\frac{f_{00}(\boldsymbol{k})}{\hbar \omega_{\boldsymbol{k}}},
$$

the interaction with the lattice modes reads

$$
\begin{aligned}
H_{\mathrm{c}-\mathrm{ph}}= & |2\rangle\langle 2| \sum_{\boldsymbol{k}} F_{22}(\boldsymbol{k})\left(b_{\boldsymbol{k}}+b_{-\boldsymbol{k}}^{\dagger}\right) \\
& +\left[|\tilde{1}\rangle\langle 2| \sum_{\boldsymbol{k}} F_{12}(\boldsymbol{k})\left(b_{\boldsymbol{k}}+b_{-\boldsymbol{k}}^{\dagger}\right)+\text { H.c. }\right],
\end{aligned}
$$

where $F_{22}(\boldsymbol{k})=f_{22}(\boldsymbol{k})-f_{00}(\boldsymbol{k})$ and $F_{12}(\boldsymbol{k})=$ $f_{12}(\boldsymbol{k}) e^{-i\left(\omega_{1} t-\gamma_{0}\right)}$. Additionally, there is a polaron-like energy shift which is included in the control Hamiltonian $H_{\mathrm{C}}$. The interband off-diagonal phonon coupling [the second term in Eq. (3)] has a negligible effect due to energetic reasons 17 and can be disregarded. For spectrally narrow pulses, which are needed for the adiabaticity of the procedure, only acoustic phonons contribute to the dephasing. For overlapping electron and hole wave functions, the excitation of a confined trion does not involve considerable charge redistribution and the effect of the piezoelectric coupling is very weak ${ }^{25}$. Therefore, we consider only the interaction with longitudinal acoustic phonons via the deformation potential coupling.

We assume for simplicity that the trion state is described by a product of electron and hole wave functions $\Psi_{\mathrm{e}(\mathrm{h})}(\boldsymbol{r})$ which are the same as those corresponding to a single confined carrier. This is a reasonable approximation in the strong confinement limit, where the Coulomb interaction is of minor influence on the wave functions and leads only to energy renormalization effects which are included in the transition energies. The coupling between the trion and phonons [the first term in Eq. (3)] has the form (see Appendix A)

$$
F_{22}(\boldsymbol{k})=f_{22}(\boldsymbol{k})-f_{00}(\boldsymbol{k})=\sqrt{\frac{\hbar k}{2 \rho V c_{1}}}\left(D_{\mathrm{e}}-D_{\mathrm{h}}\right) \mathcal{F}(\boldsymbol{k}),
$$

where $\rho$ is the crystal density, $V$ is the normalization volume of the phonon modes, $c_{1}$ is the longitudinal speed of sound, and $D_{\mathrm{e}(\mathrm{h})}$ is the deformation potential constant for the electron (hole). The form factor $\mathcal{F}(\boldsymbol{k})$ depends on the geometry of the wave functions $\Psi_{\mathrm{e}(\mathrm{h})}(\boldsymbol{r})$. We assume Gaussian wave functions

$$
\Psi_{\mathrm{e}(\mathrm{h})}(\boldsymbol{r}) \sim \exp \left(-\frac{r_{\perp}^{2}}{2 l_{\mathrm{e}(\mathrm{h})}^{2}}-\frac{z^{2}}{2 l_{z}^{2}}\right),
$$

where $l_{\mathrm{e}(\mathrm{h})}$ is the confinement size for an electron (a hole) in the $x y$ plane, $l_{z}$ is the common confinement size along $z$, and $r_{\perp}$ and $r_{z}$ are the corresponding components of the position. Then, neglecting the small correction resulting from the difference between the electron and hole confinement sizes, one finds

$$
\mathcal{F}(\boldsymbol{k})=e^{-\left(k_{\perp} l / 2\right)^{2}-\left(k_{z} l_{z} / 2\right)^{2}},
$$

where $l^{2}=\left(l_{\mathrm{e}}^{2}+l_{\mathrm{h}}^{2}\right) / 2$ and $k_{\perp}$ and $k_{z}$ are the components of the wave vector in the $x y$ plane and along $z$, respectively (see Appendix $₫$ for details).

The carrier-photon interaction Hamiltonian in the rotating wave approximation reads (in the rotating frame)

$$
\begin{aligned}
H_{\mathrm{c}-\mathrm{rad}}= & \frac{1}{\sqrt{2}} \sum_{\mathbf{q}, \lambda} g_{\mathbf{q} \lambda} c_{\mathbf{q} \lambda}^{\dagger}\left[e^{i \omega_{0} t}|\tilde{0}\rangle\langle 2|\right. \\
& \left.+e^{i\left(\omega_{1} t-\gamma\right)}|\tilde{1}\rangle\langle 2|\right]+ \text { H.c. }
\end{aligned}
$$




\begin{tabular}{lll}
\hline Deformation potential coupling & $D_{\mathrm{e}}-D_{\mathrm{h}} 8 \mathrm{eV}$ \\
Crystal density & $\rho$ & $5360 \mathrm{~kg} / \mathrm{m}^{3}$ \\
Speed of sound (longitudinal) & $c_{\mathrm{l}}$ & $5150 \mathrm{~m} / \mathrm{s}$ \\
Wave function width in-plane & $l$ & $5 \mathrm{~nm}$ \\
Wave function width in $z$ direction & $l_{z}$ & $1 \mathrm{~nm}$ \\
Trion decay rate & $\Gamma$ & $1 \mathrm{~ns}^{-1}$ \\
Zeeman splitting & $\hbar \Delta_{\mathrm{B}}$ & $1 \mathrm{meV}$ \\
\hline
\end{tabular}

TABLE I: System parameters used in the calculations.

with the coupling constants

$$
g_{\mathbf{q} \lambda}=-i \sum_{\alpha=1}^{3} d_{\alpha} \sqrt{\frac{\hbar \omega_{\mathbf{q}}^{\prime}}{2 \epsilon_{0} \epsilon_{\mathrm{r}} V}} e_{\alpha}^{(\lambda)}(\mathbf{q}) .
$$

Here, $\alpha$ denotes Cartesian components, $d_{\alpha}$ is the interband dipole moment, $\epsilon_{0}$ and $\epsilon_{\mathrm{r}}=n_{\mathrm{r}}^{2}$ are the vacuum dielectric constant and the semiconductor relative dielectric constant, respectively, and $e^{(\lambda)}(\mathbf{q})$ is the unit polarization vector.

In Tab. I, the material parameters (corresponding to a self-assembled InAs/GaAs system) are given.

\section{UNPERTURBED SPIN ROTATION}

In this section, we present the formal description of the spin rotation procedure ${ }^{14}$ without the interaction with the environment. In the ideal case, the evolution is slow and may be described by invoking the adiabatic theorem 26 .

In the considered three-level system [Fig. 1(b)], it is possible to perform an arbitrary rotation of the electron spin via the intermediate trion state $|2\rangle$. To show this, one sets in the control Hamiltonian $H_{\mathrm{C}}$ [Eq. (1)]

$$
\Delta=\Theta(t) \cos [2 \phi(t)]
$$

and

$$
\Omega_{0}(t)=\Omega(t) \cos \beta, \quad \Omega_{1}(t)=\Omega(t) \sin \beta,
$$

where

$$
\Omega(t)=\Theta(t) \sin [2 \phi(t)]
$$

Here, $\Theta(t)=\sqrt{\Omega^{2}(t)+\Delta^{2}}$ is the (time-dependent) effective Rabi frequency and

$$
\sin ^{2} \phi(t)=\frac{1}{2}\left(1-\frac{\Delta}{\sqrt{\Omega^{2}(t)+\Delta^{2}}}\right) .
$$

For $\Delta>0$, switching the pulses off corresponds to $\phi \rightarrow 0$.

To ensure an adiabatic evolution, the angle $\beta$, defined via $\tan \beta=\Omega_{1} / \Omega_{0}$, should vary slowly in time. We choose $\Omega_{0}$ and $\Omega_{1}$ to have the same envelope shapes, so that $\beta$ becomes time independent.
We introduce new basis states

$$
\begin{aligned}
& |B\rangle=e^{i \gamma} \cos \beta|\tilde{0}\rangle+\sin \beta|\tilde{1}\rangle, \\
& |D\rangle=e^{i \gamma} \sin \beta|\tilde{0}\rangle-\cos \beta|\tilde{1}\rangle
\end{aligned}
$$

which are superpositions of the qubit states $|0\rangle$ and $|1\rangle$ selected by the laser pulses, where only the bright state $|B\rangle$ is coupled to the trion state and the orthogonal dark state $|D\rangle$ remains unaffected. The Hamiltonian $H_{\mathrm{C}}$ which generates the ideal evolution [Eq. (11)] now reads

$$
H_{\mathrm{C}}=\hbar \Delta|2\rangle\langle 2|+\frac{\hbar}{2} \Omega(t)(|B\rangle\langle 2|+| 2\rangle\langle B|)
$$

and has the instantaneous eigenstates

$$
\begin{aligned}
& \left|a_{0}(t)\right\rangle=|D\rangle \\
& \left|a_{1}(t)\right\rangle=\cos \phi(t)|B\rangle-\sin \phi(t)|2\rangle \\
& \left|a_{2}(t)\right\rangle=\sin \phi(t)|B\rangle+\cos \phi(t)|2\rangle
\end{aligned}
$$

with the corresponding eigenvalues

$$
\begin{aligned}
& \lambda_{0}(t)=0, \\
& \lambda_{1}(t)=-\hbar \Theta(t) \sin ^{2} \phi(t)=\frac{\hbar}{2}\left(\Delta-\sqrt{\Delta^{2}+\Omega^{2}(t)}\right), \\
& \lambda_{2}(t)=\hbar \Theta(t) \cos ^{2} \phi(t)=\frac{\hbar}{2}\left(\Delta+\sqrt{\Delta^{2}+\Omega^{2}(t)}\right) .
\end{aligned}
$$

The system evolution is realized by the change of the so called tipping angle $\phi(t)$ [see Fig. 2(a)], i.e., by the change of the pulse amplitudes. The condition for adiabaticity is a slow change of $\phi(t)$ in comparison with the rate of the adiabatic motion given by the effective Rabi frequency, $\mid \dot{\phi(} t) \mid \ll \Theta(t)$. If the adiabatic condition is met, the state of the system (initially a combination of $|0\rangle$ and $|1\rangle$ ) remains in the subspace spanned by the two eigenstates $\left|a_{0}\right\rangle$ and $\left|a_{1}\right\rangle$ during the whole process.

The evolution operator $U_{\mathrm{C}}(t)$ in the absence of the environment perturbation (in the basis $|D\rangle,|B\rangle,|2\rangle$ ) has the form

$$
U_{\mathrm{C}}(t)=\left(\begin{array}{ccc}
1 & 0 & 0 \\
0 & e^{-i \Lambda_{1}(t)} \cos \phi(t) & e^{-i \Lambda_{2}(t)} \sin \phi(t) \\
0 & -e^{-i \Lambda_{1}(t)} \sin \phi(t) & e^{-i \Lambda_{2}(t)} \cos \phi(t)
\end{array}\right),
$$

where

$$
\Lambda_{n}(t)=\frac{1}{\hbar} \int_{t_{0}}^{t} d \tau \lambda_{n}(\tau), \quad(n=1,2) .
$$

From Eq. (9), it is clear that after the operation, when the pulses are switched off $(\phi \rightarrow 0)$, an arbitrary initial electron spin state will have acquired a phase in the $|B\rangle$ component with respect to the orthogonal dark superposition $|D\rangle$. The resulting unitary transformation in the qubit subspace can be written as

$$
U_{\mathrm{C}}(\infty)=e^{-\frac{i}{2} \Lambda_{1} \vec{\sigma} \cdot \vec{n}}=\cos \frac{\Lambda_{1}}{2} \mathbf{I}-i \sin \frac{\Lambda_{1}}{2} \vec{\sigma} \cdot \vec{n},
$$



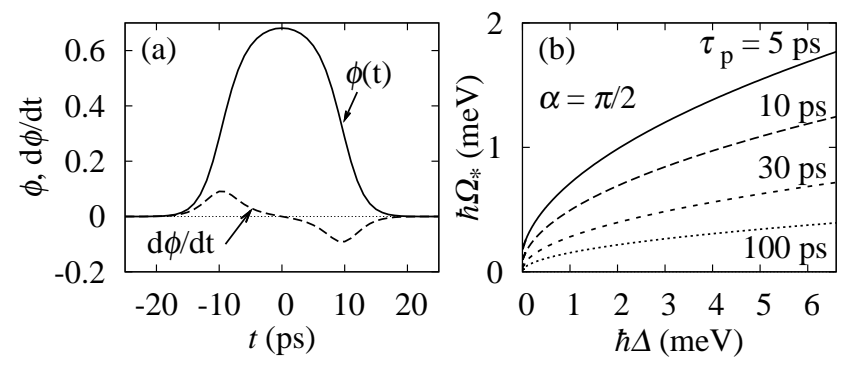

FIG. 2: (a) The tipping angle and its derivative as a function of time for a detuning $\hbar \Delta=1 \mathrm{meV}$ and a pulse duration $\tau_{p}=5$ ps. (b) The adjusted amplitude of the control pulse for the $\pi / 2$ rotation about the $z$ axis as a function of detuning for different fixed pulse durations.

where $\mathbf{I}$ is the unit operator and $\vec{\sigma}$ is the vector of Pauli matrices in the original qubit basis $|0\rangle,|1\rangle$. This transformation corresponds to a rotation through an angle $\Lambda_{1}(\infty)$ about the axis $\vec{n}$,

$$
\vec{n}=[-\cos \gamma \sin (2 \beta), \sin \gamma \sin (2 \beta),-\cos (2 \beta)],
$$

which depends on the ratio of the pulse envelopes and on the relative phase of the pulses.

For convenience, we write the general initial state of the spin qubit in the form

$$
\left|\psi_{0}\right\rangle=\cos \frac{\vartheta}{2}|B\rangle+e^{i \varphi} \sin \frac{\vartheta}{2}|D\rangle,
$$

where $\vartheta$ and $\varphi$ are angles on a Bloch sphere. We will also need two states orthogonal to $\left|\psi_{0}\right\rangle$, which we choose in the form

$$
\left|\psi_{1}\right\rangle=\sin \frac{\vartheta}{2}|B\rangle-e^{i \varphi} \cos \frac{\vartheta}{2}|D\rangle, \quad\left|\psi_{2}\right\rangle=|2\rangle .
$$

We assume that the rotation is performed using Gaussian control pulses

$$
\Omega(t)=\Omega_{*} \exp \left(-\frac{t^{2}}{2 \tau_{\mathrm{p}}^{2}}\right),
$$

where $\Omega_{*}$ is the amplitude of the control pulse and $\tau_{\mathrm{p}}$ its duration. In the following discussion, we will treat the pulse duration $\tau_{\mathrm{p}}$ and the detuning $\Delta$ as tunable parameters, while the pulse amplitude $\Omega_{*}$ will be adjusted to achieve the desired rotation of the qubit. The amplitude as a function of detuning for a $\pi / 2$ rotation about the $z$ axis [growth direction, see Fig. 1(a)] is plotted for different pulse durations in Fig. 2(b).

\section{IMPERFECTIONS OF THE UNITARY EVOLUTION}

Before we study the impact of the environment, let us discuss the limitations imposed on the driving by the re- quirement of adiabatic evolution, as well as the effect of the oscillatory terms contained in $H_{\mathrm{C}}^{\prime}$ in Eq. (11) and neglected in the discussion presented in the previous section (the former has also been studied in Ref. 19).

A perfectly adiabatic evolution does not transfer the qubit states $|0\rangle$ and $|1\rangle$ into the trion state $|2\rangle$, which is only slightly occupied during the gating. In realistic experiments, the parameters cannot be changed infinitely slowly, so that there is a nonzero probability of a jump from the ideal instantaneous (adiabatic) state to one of the other states. Representing the exact system state in terms of the adiabatic eigenstates [Eqs. $[8 \mathrm{ar}$ - $)]$,

$$
|\psi\rangle=\sum_{n} c_{n}(t) e^{-i \Lambda_{n}(t)}\left|a_{n}(t)\right\rangle
$$

one finds the equation for the probability amplitudes 26 ,

$$
\dot{c}_{m}(t)=-\sum_{n} e^{i\left[\Lambda_{m}(t)-\Lambda_{n}(t)\right]}\left\langle a_{m}(t) \mid \dot{a}_{n}(t)\right\rangle c_{n}(t) .
$$

The state $\left|a_{0}\right\rangle$ is time independent and represents the dark state $|D\rangle$ decoupled from the trion state $|2\rangle$. Moreover, $\left\langle a_{0}(t) \mid \dot{a}_{1}(t)\right\rangle=0$. Therefore, to the leading order, the only unwanted transition is to the state $\left|a_{2}\right\rangle$, which becomes the trion state after switching off the pulses. The corresponding amplitude is

$$
c_{2}^{\mathrm{na}} \approx-\int_{-\infty}^{\infty} d t e^{i\left[\Lambda_{2}(t)-\Lambda_{1}(t)\right]}\left\langle a_{2}(t) \mid \dot{a}_{1}(t)\right\rangle c_{1}(0)
$$

If the initial state is $\left|\varphi_{0}(t)\right\rangle$, as given by Eq. (10), then $c_{1}(0)=\cos (\vartheta / 2)$ and

$$
c_{2}^{\mathrm{na}} \approx \cos \frac{\vartheta}{2} \int_{-\infty}^{\infty} d t e^{i\left[\Lambda_{2}(t)-\Lambda_{1}(t)\right]} \dot{\phi}(t) .
$$

The other source of imperfections in the controlled evolution are the rotating (off-resonant) terms contained in the Hamiltonian $H_{\mathrm{C}}^{\prime}$, which reads in the new basis

$$
\begin{aligned}
& H_{\mathrm{C}}^{\prime}= \\
& \quad \frac{\hbar}{2} \Omega(t)\left[e^{i\left(\Delta_{\mathrm{B}} t-\gamma\right)} \sin ^{2} \beta-e^{-i\left(\Delta_{\mathrm{B}} t-\gamma\right)} \cos ^{2} \beta\right]|D\rangle\langle 2| \\
& \quad+\frac{\hbar}{2} \Omega(t) \cos \left(\Delta_{\mathrm{B}} t-\gamma\right) \sin (2 \beta)|B\rangle\langle 2|+\text { H.c. }
\end{aligned}
$$

We assume that the correction to the unitary evolution resulting from these terms is small and treat them perturbatively. The effects of the additional Hamiltonian $H_{\mathrm{C}}^{\prime}$ may be of two kinds: additional unitary rotation within the computational space and leakage to the trion state. The former can be taken into account when designing the control pulses and compensated by a suitable modification of the control parameters. Therefore, we treat only the latter as an error. The amplitude for the trion excitation is given by 


$$
\begin{aligned}
c_{2}^{\text {off }}= & \int_{-\infty}^{\infty} d t\left\langle 2\left|H_{\mathrm{C}}^{\prime}(t)\right| \psi_{0}\right\rangle= \\
& \frac{1}{2} \cos \frac{\vartheta}{2} \sin (2 \beta) \int_{-\infty}^{\infty} d t e^{i\left[\Lambda_{2}(t)-\Lambda_{1}(t)\right]} \Omega(t) \cos \left(\Delta_{\mathrm{B}} t-\gamma\right) \cos [2 \phi(t)] \\
& +\frac{1}{2} e^{i \varphi} \sin \frac{\vartheta}{2} \int_{-\infty}^{\infty} d t e^{i \Lambda_{2}(t)} \Omega(t) \cos \phi(t)\left[e^{-i\left(\Delta_{\mathrm{B}} t-\gamma\right)} \sin ^{2} \beta-e^{i\left(\Delta_{\mathrm{B}} t-\gamma\right)} \cos ^{2} \beta\right],
\end{aligned}
$$

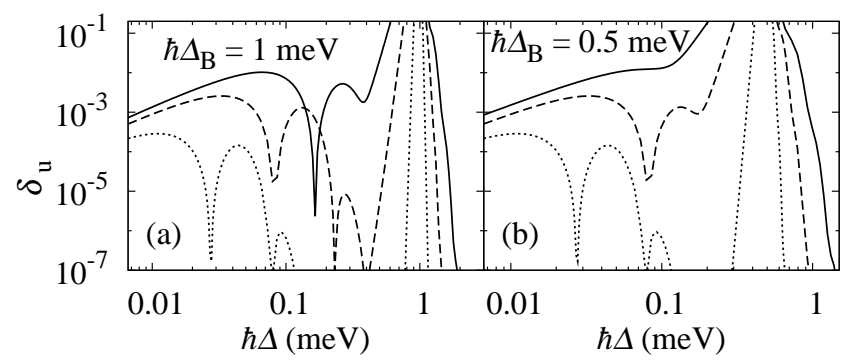

FIG. 3: The error due to the unitary corrections as a function of detuning for two values of the Zeeman splitting $\Delta_{\mathrm{B}}$ and three values of the pulse duration: $\tau_{\mathrm{p}}=5 \mathrm{ps}$ (solid lines), $\tau_{\mathrm{p}}=10 \mathrm{ps}$ (dashed lines), and $\tau_{\mathrm{p}}=30 \mathrm{ps}$ (dotted lines).

where $H_{\mathrm{C}}^{\prime}(t)=U_{\mathrm{C}}^{\dagger}(t) H_{\mathrm{C}}^{\prime} U_{\mathrm{C}}(t)$ is the relevant Hamiltonian in the interaction picture with respect to the perfect evolution described by the evolution operator in Eq. (9).

The error due to both unitary corrections described above is given by the total probability of leakage to the trion state,

$$
\delta_{\mathrm{u}}=\left|c_{2}^{\mathrm{na}}+c_{2}^{\mathrm{off}}\right|^{2},
$$

and is plotted in Fig. 3 for a $\pi / 2$ rotation about the $z$ axis as a function of detuning for two values of the Zeeman splitting and different pulse durations. If the values of the detuning approach the Zeeman splitting $\Delta_{\mathrm{B}}$, the error becomes very large, since one spin state is then almost resonantly coupled to the trion state. This induces a large trion occupation which inhibits a coherent spin rotation in this scheme. If the evolution is fast (short pulse durations $\tau_{\mathrm{p}}$ ), the adiabatic condition is not met for small detunings, which results in larger errors.

In addition, the unitary error shows oscillations, visible in Figs. B $3(a, b)$, which are due to the nonadiabatic contribution. In order to understand their origin, let us note that, in spite of the smooth Gaussian pulse envelope, the tipping angle evolves in a step-wise manner [see Fig. 2(a)] (especially for strong pulses). Thus, $\dot{\phi}(t)$ has two peaks of opposite signs at $t= \pm t_{1}$, where $t_{1}$ is a certain time, depending on the pulse duration $\tau_{\mathrm{p}}\left(t_{1} \approx 10 \mathrm{ps}\right.$ in Fig. 2(a)]). Hence, according to Eq. (11), the probability for a non-adiabatic jump is approximately proportional to $\sin ^{2}\left[\Lambda_{2}\left(t_{1}\right)-\Lambda_{1}\left(t_{1}\right)-\Lambda_{2}\left(-t_{1}\right)+\Lambda_{1}\left(-t_{1}\right)\right]$ and is therefore an oscillating function of the control param- eters. This oscillation of the transition probability reflects the interference of the amplitudes for non-adiabatic jumps when the trion is switched on and off.

For reasonably long pulses, one can indicate two regimes of the detuning where the operation on the qubit has a high fidelity. The values of the detuning have to be chosen either above the Zeeman splitting or below it and larger than $0.1 \mathrm{meV}$. To take advantage of the available fast optical control methods and insure the adiabaticity of the evolution, one can perform the operation with short pulse durations of several picoseconds and detunings of a few meV.

\section{ENVIRONMENT PERTURBATION DURING OPERATION ON THE QUBIT}

In this section, we summarize the general method for describing the effects of a perturbation due to the environment on an arbitrary operation on a qubit. A full account of this approach is given in earlier works 17,18 . Here, we give a brief overview for the sake of completeness and for reference in the following sections.

The evolution in the absence of the perturbation due to the environment is described by the unitary evolution operator $U_{0}(t)=U_{\mathrm{C}}(t) \otimes e^{-i H_{\text {env }} t}$. The effect of the interaction with the environment is calculated using the secondorder Born expansion of the evolution equation for the density matrix. We include the effect of the driving field non-perturbatively and treat the carrier-environment interaction within a perturbation theory.

The reduced density matrix of the qubit reads

$$
\rho(t)=U_{0}(t)\left[\rho_{0}+\rho^{(2)}(t)\right] U_{0}^{\dagger}(t),
$$

where $\rho^{(2)}(t)$ is the correction to the density matrix resulting from the interaction with the environment (in the interaction picture) and $\rho_{0}$ the initial state of the qubit, which is assumed to be pure, $\rho_{0}=\left|\psi_{0}\right\rangle\left\langle\psi_{0}\right|$. The initial state of the system (qubit together with the environment) has the form $\rho_{0} \otimes \rho_{T}$, where $\rho_{T}$ is the thermal equilibrium state of the environment bath.

To quantify the quality of the operation on a qubit, we use the fidelity 27

$$
F=\left\langle\psi_{0}\left|U_{0}^{\dagger}(\infty) \rho(\infty) U_{0}(\infty)\right| \psi_{0}\right\rangle^{1 / 2},
$$


which is a measure of the overlap between the ideal (pure) final state without perturbation, $U_{0}(\infty)\left|\psi_{0}\right\rangle$, and the actual final state of the system given by the density matrix $\rho(\infty)$. If the procedure is performed ideally, i.e. without discrepancies from the desired qubit operation, then $F=1$. The fidelity loss $\delta=1-F^{2}$ is referred to as the error of the quantum gate. Inserting Eq. (12) into Eq. (13), with $\rho_{0}=\left|\psi_{0}\right\rangle\left\langle\psi_{0}\right|$, one finds

$$
\delta=-\left\langle\psi_{0}\left|\rho^{(2)}(\infty)\right| \psi_{0}\right\rangle .
$$

The density matrix correction is calculated from a perturbation expansion ${ }^{28}$ :

$$
\rho^{(2)}(t)=-\frac{1}{\hbar^{2}} \int_{t_{0}}^{t} d \tau \int_{t_{0}}^{\tau} d \tau^{\prime} \operatorname{Tr}_{\mathrm{R}}\left[V(\tau),\left[V\left(\tau^{\prime}\right), \rho_{0}\right]\right],
$$

where $\operatorname{Tr}_{R}$ is the trace with respect to the reservoir degrees of freedom and $V(t)=U_{0}^{\dagger}(t) V U_{0}(t)$ is the carrierenvironment Hamiltonian in the interaction picture. It can always be written in the general form

$$
V=\sum_{n n^{\prime}} S_{n n^{\prime}} \otimes R_{n n^{\prime}}
$$

where $S_{n n^{\prime}}$ acts on the carrier subsystem, $R_{n n^{\prime}}$ acts on the environment of phonons or photons, and $R_{n n^{\prime}}=$ $R_{n^{\prime} n}^{\dagger}, S_{n n^{\prime}}=S_{n^{\prime} n}^{\dagger}$. It is easy to see that Eqs. (2) and (7) have this structure.

It is convenient to introduce two sets of spectral functions. The first is a family of spectral densities of the reservoir (phonons or photons), defined as

$$
R_{n n^{\prime}, m m^{\prime}}(\omega)=\frac{1}{2 \pi} \int d t e^{i \omega t}\left\langle R_{n n^{\prime}}(t) R_{m m^{\prime}}\right\rangle,
$$

with the operator $R_{n n^{\prime}}$ transformed into the interaction picture $R_{n n^{\prime}}(t)=U_{0}^{\dagger}(t) R_{n n^{\prime}} U_{0}(t)$. The functions from the second set are nonlinear spectral characteristics of the driven evolution,

$$
S_{n n^{\prime}, m m^{\prime}}(\omega)=\sum_{i}\left\langle\psi_{0}\left|Y_{n^{\prime} n}^{\dagger}(\omega)\right| \psi_{i}\right\rangle\left\langle\psi_{i}\left|Y_{m m^{\prime}}\right| \psi_{0}\right\rangle,
$$

where $\left|\psi_{i}\right\rangle$ span the subspace orthogonal to the initial state $\left|\psi_{0}\right\rangle$ and

$$
Y_{n n^{\prime}}(\omega)=\int d t S_{n n^{\prime}}(t) e^{-i \omega t},
$$

with $S_{n n^{\prime}}(t)=U_{0}^{\dagger}(t) S_{n n^{\prime}} U_{0}(t)$. The various terms in Eq. (16) describe transitions to different states orthogonal to the desired state $\left|\psi_{0}\right\rangle$. In the long time limit for a time-independent system, all of them either vanish or turn into energy-conserving Dirac delta functions, restoring Fermi's golden rule for transition probabilities 29 . In the general case, they are broadened due to time dependence.
Using the definitions in Eqs. (15) and (16), the error [Eq. (14)] can be written in the form ${ }^{17}, 18$

$$
\delta=\sum_{n n^{\prime}, m m^{\prime}} \int d \omega R_{n n^{\prime}, m m^{\prime}}(\omega) S_{n n^{\prime}, m m^{\prime}}(\omega) .
$$

The error can thus be expressed as an overlap between spectral functions, which are the "building blocks" for the calculation of the environment effects on the quantum evolution. A detailed derivation of Eq. (18) can be found in Refs. 17 and 18 .

The perturbative approach described above obviously yields only an approximate description of decoherence. In the case of phonon-induced dephasing, comparisons with exact results (for ultrashort laser pulses) ${ }^{30}$ and with correlation expansion results 31 show that the perturbative results are very accurate as long as the overall dephasing effect is weak: the inaccuracy is of the order of $\delta^{2}$, where $\delta$ is some measure of decoherence, e.g., the fidelity loss, Eq. (18). The same holds for the decoherence induced by the radiative decay of the trion, as we show in Appendix B.

Thus, to calculate the error of the quantum gate due to the interaction with the environment, one needs to derive the two spectral functions. These will yield a transparent spectral interpretation for the various contributions to the qubit dephasing and provide a possibility to seek the optimal conditions depending on the system properties.

\section{DECOHERENCE MECHANISMS}

In this section, the different kinds of decoherence mechanisms (the coupling of the carriers to phonons and photons) are studied. We apply the general theory introduced in the former section to calculate the total error of a spin rotation through an angle of $\pi / 2$ about the $z$ axis. The quantitative estimates are given for charged self-assembled InAs/GaAs quantum dot.

\section{A. Interaction with the phonons}

It follows from Eq. (3) that the carrier-phonon interaction is described by just one pair of operators, $S_{22}=|2\rangle\langle 2|$ and $R_{22}=\sum_{\boldsymbol{k}} F_{22}(\boldsymbol{k})\left(b_{\boldsymbol{k}}+b_{-\boldsymbol{k}}^{\dagger}\right)$. Therefore, one needs only two spectral functions: the spectral density of the phonon reservoir $R_{22,22}(\omega) \equiv R_{\mathrm{ph}}(\omega)$ and the spectral characteristics of the driving $S_{22,22}(\omega) \equiv S_{\mathrm{ph}}(\omega)$ to calculate the phonon-induced error

$$
\delta_{\mathrm{ph}}=\int d \omega R_{\mathrm{ph}}(\omega) S_{\mathrm{ph}}(\omega) .
$$

Using Eq. (15), one finds the explicit form of the former:

$$
\begin{aligned}
& R_{\mathrm{ph}}(\omega)= \\
& \frac{1}{\hbar^{2}}\left[n_{\mathrm{B}}(\omega)+1\right] \sum_{\boldsymbol{k}}\left|F_{22}(\boldsymbol{k})\right|^{2}\left[\delta\left(\omega-\omega_{\boldsymbol{k}}\right)+\delta\left(\omega+\omega_{\boldsymbol{k}}\right)\right],
\end{aligned}
$$




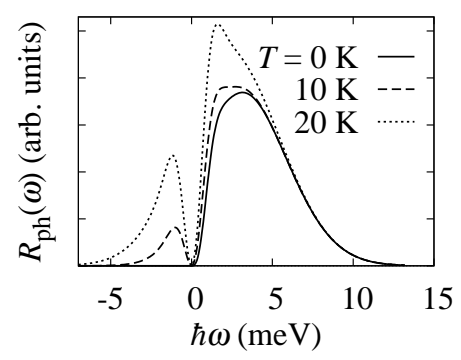

FIG. 4: Spectral density of the phonon reservoir at different temperatures $T$.

where $n_{\mathrm{B}}(\omega)$ is the Bose distribution function.

The function $R_{\mathrm{ph}}(\omega)$ (see Appendix $\mathrm{A}$ for a detailed derivation) is plotted in Fig. 4 for different temperatures $T$. The negative frequency part of the function corresponds to phonon absorption by the carriers and is nonzero only for finite temperatures, while the positive part represents the emission processes and always has finite values. The phonon spectral density has a cut-off at the frequency $\omega \approx c_{\mathrm{l}} / l$, which corresponds to the inverse of the time phonons need to traverse the quantum dot.

The carrier part of the interaction Hamiltonian $S_{\mathrm{ph}}=$ $|2\rangle\langle 2|$ leads to a spectral characteristics containing two parts corresponding to the two orthogonal states:

$$
\begin{aligned}
& S_{\mathrm{ph}}(\omega)=s_{1}^{\mathrm{ph}}(\omega)+s_{2}^{\mathrm{ph}}(\omega) \\
& =\frac{1}{4} \sin ^{2} \vartheta\left|\int d t e^{i \omega t} \sin ^{2} \phi(t)\right|^{2} \\
& \quad+\frac{1}{4} \cos ^{2} \frac{\vartheta}{2}\left|\int d t e^{i \omega t} e^{-i\left[\Lambda_{2}(t)-\Lambda_{1}(t)\right]} \sin [2 \phi(t)]\right|^{2} .
\end{aligned}
$$

The initial state, Eq. (10), can be an arbitrary superposition of the states $|0\rangle$ and $|1\rangle$, and the error depends on the choice of the initial qubit state. In order to obtain representative error evaluations, we average the error (thus, the contributing spectral function) over the angles $(\vartheta, \varphi)$ on the Bloch sphere of the initial states, according to

$$
s_{i(\mathrm{av})}(\omega)=\frac{1}{4 \pi} \int_{0}^{\pi} d \vartheta \sin \vartheta \int_{0}^{2 \pi} d \varphi s_{i}(\omega)
$$

The averaged contributions to the spectral characteristics, corresponding to the two terms in Eq. (21), read

$$
\begin{aligned}
& s_{1(\mathrm{av})}^{\mathrm{ph}}(\omega)=\frac{1}{6}\left|\int d t e^{i \omega t} \sin ^{2} \phi(t)\right|^{2}, \\
& s_{2(\mathrm{av})}^{\mathrm{ph}}(\omega)=\frac{1}{8}\left|\int d t e^{i \omega t} e^{-i\left[\Lambda_{2}(t)-\Lambda_{1}(t)\right]} \sin [2 \phi(t)]\right|^{2} .
\end{aligned}
$$

Both contributions to the phonon-induced error are independent of $\beta$.

We can derive approximate analytical formulas for the spectral characteristics under the condition that the control pulses are much smaller than the detuning $(\Omega \ll \Delta)$,

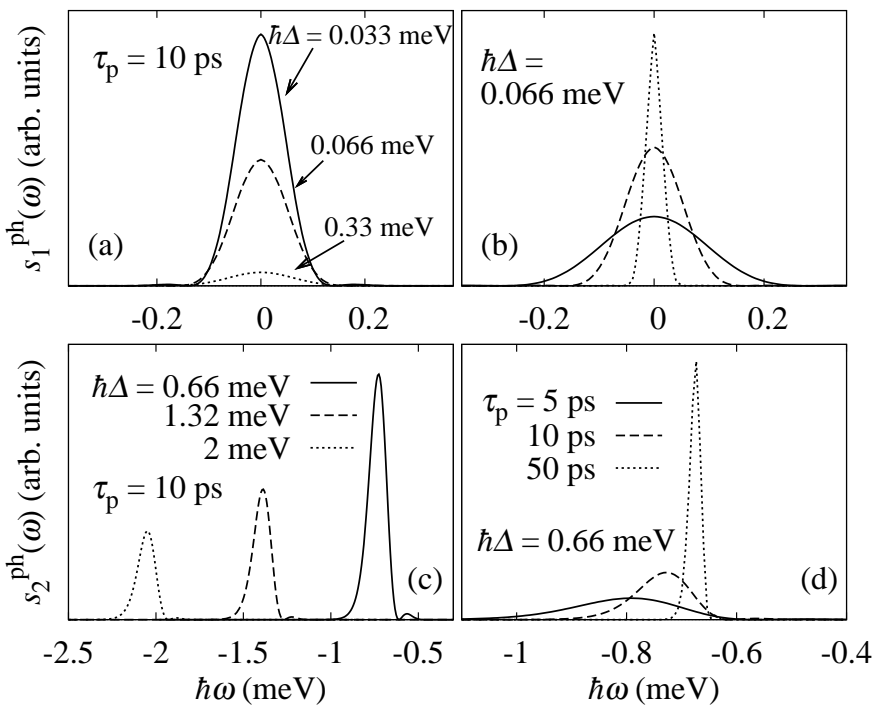

FIG. 5: (a),(b) Spectral function $s_{1}^{\mathrm{ph}}(\omega)$. (c),(d) Spectral function $s_{2}^{\mathrm{ph}}(\omega)$. In (b), (d) the line styles denote pulse durations as defined in $(\mathrm{d})$.

which is met for detunings of several meV [see Fig. 2(b)]. Thus, in this detuning regime, we obtain

$$
\begin{aligned}
& s_{1}^{\mathrm{ph}}(\omega) \approx \frac{\pi}{96} \frac{\Omega_{*}^{4} \tau_{\mathrm{p}}^{2}}{\Delta^{4}} \exp \left(-\frac{1}{2} \tau_{\mathrm{p}}^{2} \omega^{2}\right) \\
& s_{2}^{\mathrm{ph}}(\omega) \approx \frac{\pi}{4} \frac{\Omega_{*}^{2} \tau_{\mathrm{p}}^{2}}{\Delta^{2}}\left\{\exp \left[-\frac{1}{2} \tau_{\mathrm{p}}^{2}(\Delta+\omega)^{2}\right]\right. \\
&\left.\quad-\frac{\Omega_{*}^{2}}{2 \sqrt{3} \Delta^{2}} \exp \left[-\frac{1}{6} \tau_{\mathrm{p}}^{2}(\Delta+\omega)^{2}\right]\right\}^{2} .
\end{aligned}
$$

The symmetric function $s_{1}^{\mathrm{ph}}(\omega)$ [see Fig. $[5(\mathrm{a}, \mathrm{b})]$ is centered at $\omega=0$ and, for longer pulse durations, covers the low frequency part (broadening $\approx 1 / \tau_{\mathrm{p}}$ ). The broadening of this function is independent of the detuning, and, for a fixed $\tau_{\mathrm{p}}$, its area decreases for larger detunings. This part of the spectral characteristics corresponds to pure dephasing effects $25,31,32,33,34$. The resulting error [Eq. (19)] will decrease for longer pulse durations as well as for larger detunings.

The second part of the spectral characteristics $s_{2}^{\mathrm{ph}}(\omega)$ [see Fig. $5(\mathrm{c}, \mathrm{d})]$ is centered at $\omega \approx-\Delta$, and its area grows with time. Thus, the corresponding error contribution is proportional to the spectral density of the phonon reservoir around the frequency corresponding to the detuning of the laser frequency from the trion transition, with some broadening due to time dependence. Moreover, the error increases for longer operations. Therefore, this contribution may be interpreted as a real transition and describes the error due to phonon-assisted trion generation.

In order to see this more directly, let us note that the function $s_{2}^{\mathrm{ph}}(\omega)$ is relatively strongly localized around $\omega=-\Delta$, compared to the range of variation of $R(\omega)$. Therefore, the corresponding integral in Eq. (19) may be 
approximated by its Markovian limit,

$$
\delta_{2}^{\mathrm{ph}}=R_{\mathrm{ph}}(-\Delta) \int d \omega s_{2}^{\mathrm{ph}}(\omega) .
$$

The area of the spectral function $s_{2}^{\mathrm{ph}}(\omega)$ appearing in this formula may be calculated by noting that for any function $h(t)$, one has

$$
\begin{gathered}
\int d \omega\left|\int d t e^{i \omega t} h(t)\right|^{2}= \\
\quad \int d \omega \int d t \int d t^{\prime} e^{i \omega\left(t-t^{\prime}\right)} h^{*}(t) h\left(t^{\prime}\right) \\
\quad=2 \pi \int d t|h(t)|^{2}
\end{gathered}
$$

Thus, we find

$$
\delta_{2}^{\mathrm{ph}}=2 \pi \int d t R_{\mathrm{ph}}(-\Delta) \frac{1}{4} \cos ^{2} \frac{\vartheta}{2} \sin ^{2}[2 \phi(t)] .
$$

The expression under the integral is exactly the Fermi's golden rule formula for the probability that a transition from the state $\left|a_{1}\right\rangle$ to $\left|a_{2}\right\rangle$ will take place during the time $d t$ due to the (diagonal) carrier-phonon coupling given in Eq. (3). Since the state $\left|a_{2}\right\rangle$ becomes the trion state after the laser pulse is switched off, this process indeed represents a phonon-assisted transition to the trion state.

The resulting phonon-induced errors, averaged over all initial states, as functions of the detuning and pulse duration are shown in Fig. 6. The error due to pure dephasing $\delta_{1}^{\text {ph }}$ [corresponding to $\left.s_{1}^{\text {ph }}(\omega)\right]$ favors longer pulse durations and larger detunings and strongly depends on the temperature [Fig. 6(a,b)]. To perform the operation with an error smaller than $10^{-4}$, which allows for coherent quantum operation on a qubit, one needs a detuning larger than $0.06 \mathrm{meV}$ (at $T=5 \mathrm{~K}$ ). It is possible to avoid the pure dephasing error even for a fast evolution realized by pulse durations of several picoseconds and a detuning of $1 \mathrm{meV}$.

The contribution to the total error related to the phonon-assisted trion generation $\delta_{2}^{\text {ph }}$ [resulting from $\left.s_{2}^{\mathrm{ph}}(\omega)\right]$ has a different behavior in comparison with the previous one [Fig. $6(\mathrm{c}, \mathrm{d})]$ and depends even stronger on the temperature. At low temperatures $(T<1 \mathrm{~K})$, it decreases with growing detuning, but for higher temperatures $(T=1,5$ and $10 \mathrm{~K})$, it initially grows with detuning and later decreases for considerably large detunings. The maximum values correspond to the situation when the spectral function $s_{2}^{\mathrm{ph}}(\omega)$ covers the maximum of the phonon density, and the error vanishes if it lies beyond the phonon density cut-off. The pulse duration dependence of this error differs from the one for the pure dephasing error. For relatively large detunings $(\hbar \Delta>1 \mathrm{meV})$, this error favors shorter pulse durations, which is typical for real transitions.

In order to properly choose the conditions for the spin rotation that lead to a high fidelity of the operation, one

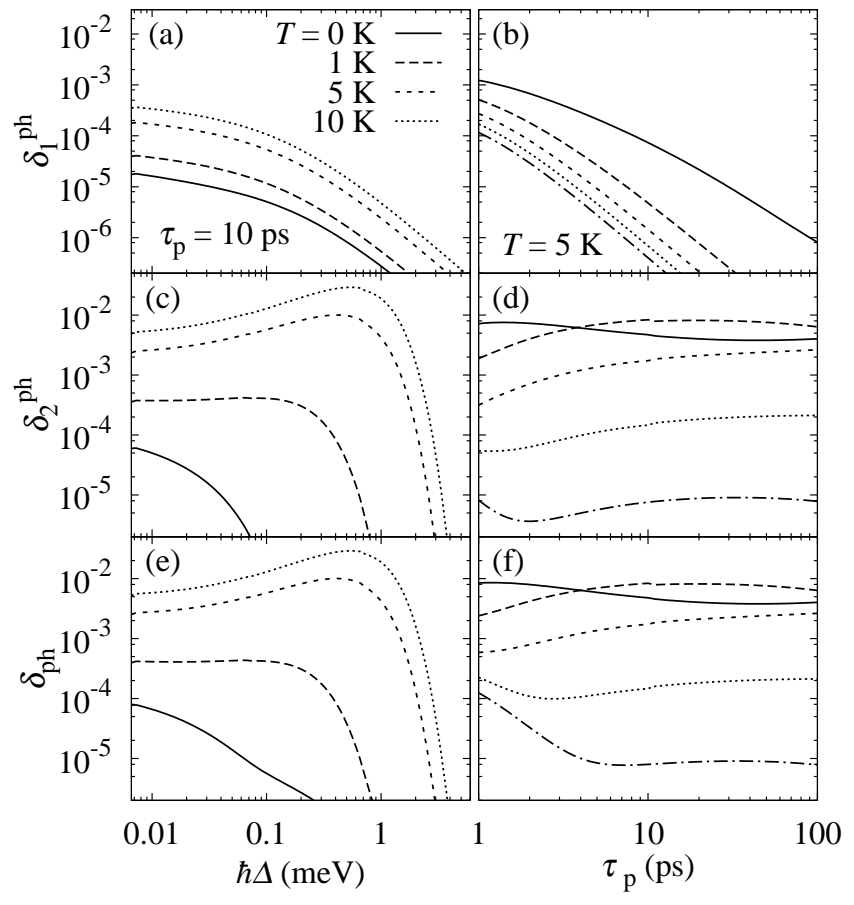

FIG. 6: (a) The dependence of the error due to pure dephasing for growing detuning for $\tau_{\mathrm{p}}=10 \mathrm{ps}$. (b) The pure dephasing error as a function of pulse duration at $T=5 \mathrm{~K}$ and for five values of detuning: $\hbar \Delta=0.066 \mathrm{meV}$ (solid line), $\hbar \Delta=0.66 \mathrm{meV}$ (longer dashed line), $\hbar \Delta=1.32 \mathrm{meV}$ (shorter dashed line), $\hbar \Delta=2 \mathrm{meV}$ (dotted line), and $\hbar \Delta=2.64 \mathrm{meV}$ (dashed-dotted line). (c), (d) As in (a), (b) but for the error due to phonon-assisted trion generation. (e), (f) As in (a), (b) but for the total error resulting from the interaction of the carriers with the phonon reservoir. In (a), (c), and (e), the line styles denote temperatures as defined in (a). In (b), (d), and (f), the lines styles denote detunings as defined in (b).

needs to take into account these two sources of error resulting from the carrier coupling to the phonon reservoir. The sum of these two errors is plotted in Fig. 6) (e,f). To achieve values of the error below $10^{-4}$ for $\tau_{\mathrm{p}}=10 \mathrm{ps}$, detunings of several $\mathrm{meV}$ are needed. For a small detuning $(\hbar \Delta=0.066 \mathrm{meV})$, the error decreases with growing pulse duration, but for larger detunings $(\hbar \Delta=0.66 \mathrm{meV}$ and $\hbar \Delta=1.32 \mathrm{meV}$ ), shorter pulse durations are more favorable. Choosing the detunings larger than several meV can suppress the influence of the phonon environment $(\hbar \Delta \gtrsim 4 \mathrm{meV}$ at $T=5 \mathrm{~K})$.

It is also possible to excite the system above the transition energies by choosing a negative detuning. In this case, the phonon-induced total error is larger in comparison with the case for positive detuning (see Fig. 17). The corresponding spectral characteristics $s_{2}^{\text {ph }}(\omega)$ is now centered at the positive frequency part of the phonon density, which has larger values than the negative part, finite even at $T=0 \mathrm{~K}$ (see Fig. (4). This corresponds to emission of a phonon, which is strongly temperature 


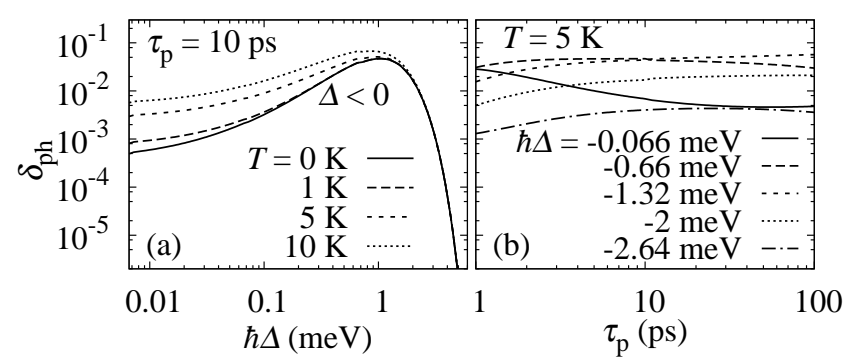

FIG. 7: The phonon-induced error for negative detunings (a) at different temperatures for growing detuning and (b) for growing pulse duration.

dependent and possible at zero temperature. Especially at low temperatures, the phonon-induced error is up to four orders of magnitude larger than the one for positive detunings. To suppress the influence of the phonon reservoir, one needs larger detunings. The dependence of this error on the pulse duration also differs. Only for small detunings $(\hbar \Delta \lesssim 1 \mathrm{meV})$, it is advantageous to use longer pulses. For relatively large detunings $(\hbar \Delta>1 \mathrm{meV})$, short pulses are favorable.

\section{B. Interaction with the photon field}

Since the considered procedure for spin rotation requires a trion occupation during the evolution, the radiative decay of the trion will result in an additional error. We calculate the effect of the photon interaction in the same manner as for the phonons including perturbatively the carrier-photon interaction.

The relevant photon energies correspond to the semiconductor band gap which is very large compared to the thermal energy. Therefore, one can use the zero temperature approximation. The carrier-photon interaction contains the following operators acting on the carrier subsystem: $S_{02}=|0\rangle\left\langle 2\left|e^{i \omega_{0} t}, S_{20}=S_{02}^{\dagger}, S_{12}=\right| 1\right\rangle\langle 2| e^{i\left(\omega_{1} t-\gamma\right)}$, and $S_{21}=S_{12}^{\dagger}$. All the resulting contributions, calculated according to the general procedure in Sec. V] can be combined into a single spectral characteristics

$$
S_{\mathrm{rad}}(\omega)=\sum_{i}\left|\left\langle\psi_{0}\left|Y_{\mathrm{rad}}^{\dagger}(\omega)\right| \psi_{i}\right\rangle\right|^{2}
$$

where

$$
\begin{aligned}
Y_{\mathrm{rad}}(\omega)= & \frac{1}{\sqrt{2}} \int d t e^{-i \omega t} U_{0}^{\dagger}(t) \\
& \times\left[(\cos \beta|B\rangle+\sin \beta|D\rangle) e^{i \omega_{0} t}\right. \\
& \left.+(\sin \beta|B\rangle-\cos \beta|D\rangle) e^{i \omega_{1} t}\right]\langle 2| U_{0}(t)
\end{aligned}
$$

and the sum is taken over all states orthogonal to the initial state $\left|\psi_{0}\right\rangle$ [Eq. (10)].

The spectral density of the photon reservoir reads 20

$$
R_{\mathrm{rad}}(\omega)=\frac{|\vec{d}|^{2} \omega^{3} n_{\mathrm{r}}}{6 \pi^{2} \hbar \epsilon_{0} c^{3}}, \quad \omega>0
$$

and the contribution to the error due to the photon interaction has the form

$$
\delta_{\mathrm{rad}}=\int d \omega R_{\mathrm{rad}}(\omega) S_{\mathrm{rad}}(\omega)
$$

Using the definition (17) and the explicit form of the evolution operator (9), one finds

$$
\left\langle\psi_{0}\left|Y_{\mathrm{rad}}^{\dagger}(\omega)\right| \psi_{i}\right\rangle=\frac{1}{\sqrt{2}} \int d t e^{i \omega t} s_{i}^{\mathrm{rad}}(t)
$$

where

$$
\begin{aligned}
& s_{1}^{\mathrm{rad}}(t)= \\
& \quad-\frac{1}{4} \sin \vartheta \sin [2 \phi(t)]\left(e^{-i \omega_{0} t} \cos \beta+e^{-i \omega_{1} t} \sin \beta\right) \\
& \quad-e^{i\left[\varphi+\Lambda_{1}(t)\right]} \cos ^{2} \frac{\vartheta}{2} \sin \phi(t)\left(e^{-i \omega_{1} t} \cos \beta-e^{-i \omega_{0} t} \sin \beta\right)
\end{aligned}
$$

and

$$
\begin{aligned}
s_{2}^{\mathrm{rad}}(t)= & -e^{-i\left[\Lambda_{2}(t)-\Lambda_{1}(t)\right]} \cos \frac{\vartheta}{2} \sin ^{2} \phi(t) \\
& \times\left(e^{-i \omega_{0} t} \cos \beta+e^{-i \omega_{1} t} \sin \beta\right) .
\end{aligned}
$$

The spectral function $S_{\text {rad }}(\omega)$ is centered at the laser frequency $\omega=\omega_{0,1} \approx 1 \mathrm{eV}$, and its width is of the order of $1 \mathrm{meV}$ or less. The photon spectral density $R_{\text {rad }}(\omega)$ [Eq. (24)] is very broad and may be assumed constant in the area of the overlap with $S_{\text {rad }}(\omega)$. Therefore, we use the Markovian approximation and obtain $\delta_{\mathrm{rad}}=R_{\mathrm{rad}}\left(\omega_{0}\right) \int d \omega S_{\mathrm{rad}}(\omega)$ with $R_{\mathrm{rad}}\left(\omega_{0}\right)=\Gamma /(2 \pi)$, where $\Gamma$ is the trion decay rate $\approx 1 \mathrm{~ns}^{-1}$. The frequency integral can be performed using Eq. (23). Upon averaging over the initial states, one obtains the resulting error due to the carrier-photon interaction: 


$$
\begin{aligned}
\delta_{\text {rad }}=\Gamma \int d t( & \left\{\frac{1}{24} \sin ^{2}[2 \phi(t)]+\frac{1}{4} \sin ^{4} \phi(t)\right\}\left\{1+\cos \left[\left(\omega_{1}-\omega_{0}\right) t\right] \sin (2 \beta)\right\} \\
& \left.+\frac{1}{3} \sin ^{2} \phi(t)\left\{1-\cos \left[\left(\omega_{1}-\omega_{0}\right) t\right] \sin (2 \beta)\right\}\right)
\end{aligned}
$$

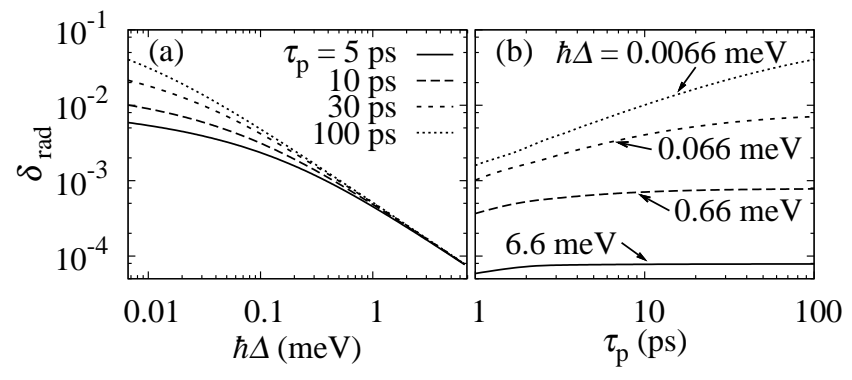

FIG. 8: The dependence of the radiative error for growing (a) detuning and (b) pulse duration.

For large enough detunings $(\Omega \ll \Delta)$, we can again obtain an approximate equation for the resulting error:

$$
\begin{aligned}
& \delta_{\text {rad }} \approx \\
& \Gamma\left(\frac{\sqrt{\pi} \Omega^{2} \tau_{\mathrm{p}}}{8 \Delta^{2}}\left\{\frac{5}{3}-\exp \left[-\frac{1}{4} \tau_{\mathrm{p}}^{2}\left(\omega_{1}-\omega_{0}\right)^{2}\right]\right\}\right. \\
&\left.\quad+\frac{\sqrt{\pi} \Omega^{4} \tau_{\mathrm{p}}}{8 \sqrt{2} \Delta^{4}}\left\{5-\exp \left[-\frac{1}{8} \tau_{\mathrm{p}}^{2}\left(\omega_{1}-\omega_{0}\right)^{2}\right]\right\}\right) .
\end{aligned}
$$

The resulting radiative error [Eq. (25)] as a function of detuning and pulse duration is plotted in Fig. 8 (a,b). This error decreases with growing detuning since the trion occupation is reduced. For small detunings, the trion occupation is relatively large since the system is excited near the resonance, and the resulting error is growing with pulse duration due to the growing probability of the radiative decay of the trion. In this regime, the error is linear in the pulse duration, $\delta_{\text {rad }} \approx \tau_{\mathrm{p}} \Gamma$. The contribution to the error due to the finite trion lifetime depends strongly on the pulse duration only for relatively small detunings. For detunings of several meV, this error is constant in time. To insure a small radiative error, one has to properly choose a large detuning, while the pulse duration can be arbitrarily short.

\section{INTERPLAY OF THE DIFFERENT KINDS OF ERRORS}

In the previous sections, we studied the detuning and pulse duration dependence of the contributions to the total error of the spin-based quantum gate. In this section, we calculate the resulting total error and discuss the interplay of and possible optimization against the constituent sources of the error.

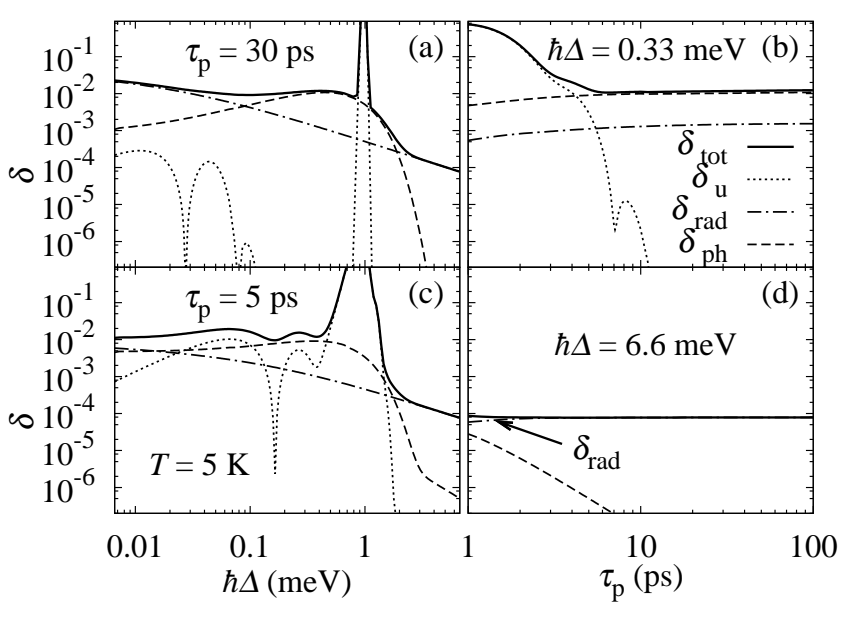

FIG. 9: Interplay of the error contributions as a function of (a), (c) detuning and (b), (d) pulse duration at $T=5 \mathrm{~K}$. Different lines represent various error sources, as defined in (b).

We start the discussion with the dependence of the contributions to the total error of the considered spin rotation on the detuning for a fixed temperature $(T=5 \mathrm{~K})$ and two pulse durations $\left(\tau_{\mathrm{p}}=30 \mathrm{ps}\right.$ and $\left.\tau_{\mathrm{p}}=5 \mathrm{ps}\right)$ [see Fig. 9(a,c)]. For small detunings, the trion occupation is large, and the dominant source of the error is the radiative decay of the trion. This contribution decreases with growing detuning, and the phonon-induced error becomes the most important source of dephasing. If the values of the detuning approach the Zeeman splitting, one spin state is almost resonantly coupled to the trion state, and the probability of the leakage to the trion state is high, which leads to a large error due to the unitary corrections, especially in the case of a short pulse $\left(\tau_{\mathrm{p}}=5 \mathrm{ps}\right)$. In the detuning regime between $0.09 \mathrm{meV}$ and $2 \mathrm{meV}$, the error resulting from the phonon coupling becomes dominant and is one order of magnitude larger than the radiative error. For detunings of several meV above the value of the cut-off of the phonon error, the only contribution which inhibits the coherent spin rotation is the trion radiative decay and has values between $10^{-3}$ and $10^{-4}$. To achieve errors smaller than $10^{-4}$, large detuning of several or tens of $\mathrm{meV}$ are needed. In this detuning regime, the limitation results from the fact that one cannot choose an arbitrarily large detuning, in particular not with a frequency corresponding to that of optical phonons, assumed to be well off-resonant in this 


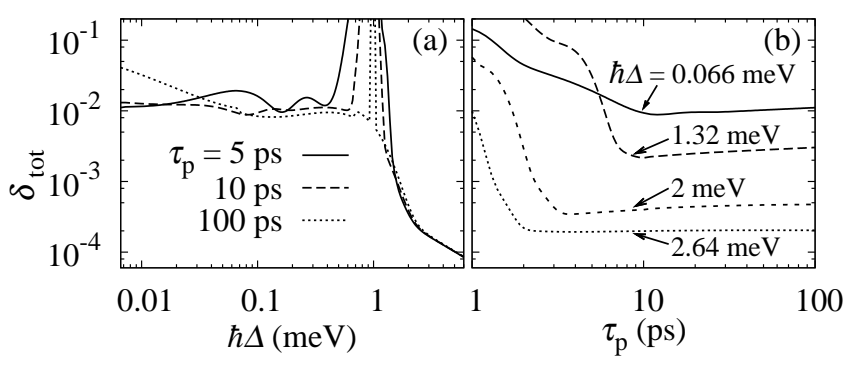

FIG. 10: The total error for growing (a) detuning and (b) pulse duration at $T=5 \mathrm{~K}$.

paper. Thus, the interplay of the different kinds of error leads to a nontrivial detuning dependence of the total error, which in general is dominated by the error due to the interaction with the phonon and photon reservoirs

Let us now discuss the dependence of the particular error contributions on the pulse duration for a fixed temperature $(T=5 \mathrm{~K})$ [see Fig. 9(b,d)]. For a small detuning $(\hbar \Delta=0.33 \mathrm{meV})$ and fast driving fields (short pulse durations of several picoseconds), the adiabatic condition is not fulfilled, and the probability of the leakage to the trion state becomes very high, which results in large errors. To minimize the influence of the errors due to the unitary corrections, one has to choose pulse durations of at least a few picoseconds. In this regime, the total error is limited by the phonon-induced contribution. The second dominant source of the error is the radiative decay of the trion. Moreover, these two contributions are almost constant in time. For a large detuning $(\hbar \Delta=6.6 \mathrm{meV})$, the evolution is perfectly adiabatic, and the error due to unitary corrections does not appear. The phonon-induced error is very small and vanishes for pulse durations of a few picoseconds, since the detuning is far above the value of the cut-off of the phonon spectral density. The only important contribution to the total error is due to the finite trion lifetime which is constant in pulse duration. One can see that it is advantageous to excite with larger detunings, but the variation of the pulse duration is not of great importance as soon as the adiabatic condition is met and the detuning is not close to the Zeeman splitting.

In order to summarize the study of the various sources of decoherence, we calculated the total error of the spinbased qubit rotation for growing detuning [Fig. 10(a)] and pulse duration [Fig. 10(b)]. For detunings smaller than $1.3 \mathrm{meV}$, the error strongly depends on the pulse duration and is relatively large. Thus, to perform a rotation with a high fidelity, detunings of several $\mathrm{meV}$ are needed. Furthermore, for such detunings, the error is constant for growing pulse duration, which opens the possibility to perform the qubit rotation with pulse durations three orders of magnitude smaller than the lifetime of the trion $\left(\Gamma^{-1} \approx 1 \mathrm{~ns}\right)$. For $\hbar \Delta \sim 5 \mathrm{meV}$, the error may be as low as $10^{-4}$ and is independent of the pulse duration.

\section{CONCLUSIONS}

We have studied a theoretical proposal of the optical control of a single spin in a single doped quantum $\operatorname{dot}^{14}$. We have investigated the sources of error of a quantum operation on a spin-based qubit and have given quantitative estimates for the implementation of spin rotation through an angle of $\pi / 2$ about the $z$ axis in a self-assembled InAs/GaAs system. The dephasing mechanisms resulting from the interaction of the carriers with phonon and photon reservoirs as well as the imperfections of the unitary evolution have been considered.

We have shown that the interplay of the constituent sources of the error leads to a nontrivial dependence of the total error on the detuning, which is in general dominated by the errors due to the coupling of the carriers to the phonon and photon environments. Furthermore, small detunings or detunings approaching the Zeeman splitting should be avoided since they lead to large trion occupations which suppress an adiabatic and coherent control. Taking into account all contributions to the total error, we showed that errors as low as $10^{-4}$ can be achieved for large detunings $(\sim 5 \mathrm{meV})$, while the pulse durations can in principle be arbitrary (but at least of a few picoseconds).

Finally, it should be noted that the calculations were performed using simple Gaussian pulses with the pulse duration and intensity as the only tunable parameters. Further reduction of the errors is very likely to be possible with pulse optimization ${ }^{30,35,36}$.

\section{Acknowledgments}

We thank C. Emary and M. Richter for fruitful discussions. A.G. acknowledges financial support from the DAAD. This work was partly supported by Grant No. N20207132/1513 of the Polish MNiSW.

\section{APPENDIX A: PHONON COUPLINGS, SPECTRAL DENSITY}

In this Appendix, we derive the effective coupling element for the carrier-phonon interaction $F_{22}(\boldsymbol{k})$ as well as the resulting spectral density of the phonon reservoir $R_{\mathrm{ph}}(\omega)$ for the studied QD system.

The general interaction Hamiltonian for confined states (restricted to the ground states of the carriers) reads

$$
\begin{aligned}
H_{\mathrm{int}}= & \sum_{\boldsymbol{k}} \sum_{\sigma}\left[D_{\mathrm{e}} \mathcal{F}_{\mathrm{e}}(\boldsymbol{k}) a_{\mathrm{e}, \sigma}^{\dagger} a_{\mathrm{e}, \sigma}-D_{\mathrm{h}} \mathcal{F}_{\mathrm{h}}(\boldsymbol{k}) a_{\mathrm{h}, \sigma}^{\dagger} a_{\mathrm{h}, \sigma}\right] \\
& \times \sqrt{\frac{\hbar k}{2 \rho V c_{\mathrm{l}}}}\left(b_{\boldsymbol{k}}+b_{-\boldsymbol{k}}^{\dagger}\right),
\end{aligned}
$$

where $a_{\mathrm{e}(\mathrm{h}), \sigma}, a_{\mathrm{e}(\mathrm{h}), \sigma}^{\dagger}$ are the annihilation and creation operators, respectively, for an electron or a hole in the con- 
fined ground state with spin $\sigma$, and the form factors are given by

$$
\mathcal{F}_{\mathrm{e}(\mathrm{h})}(\boldsymbol{k})=\int_{-\infty}^{+\infty} d^{3} \boldsymbol{r} \Psi_{\mathrm{e}(\mathrm{h})}^{*}(\boldsymbol{r}) e^{i \boldsymbol{k} \boldsymbol{r}} \Psi_{\mathrm{e}(\mathrm{h})}(\boldsymbol{r})=\mathcal{F}_{\mathrm{e}(\mathrm{h})}^{*}(-\boldsymbol{k}) .
$$

For Gaussian wave functions as in Eq. (5), one explicitly finds by a simple integration

$$
\mathcal{F}_{\mathrm{e}(\mathrm{h})}(\boldsymbol{k})=\exp \left(-\frac{1}{4} k_{\perp}^{2} l_{\mathrm{e}(\mathrm{h})}^{2}-\frac{1}{4} k_{z}^{2} l_{z}^{2}\right)
$$

For a single electron state $|0\rangle=a_{\mathrm{e}, \uparrow}^{\dagger}|\mathrm{vac}\rangle$ or $|1\rangle=$ $a_{\mathrm{e}, \downarrow}^{\dagger}|\operatorname{vac}\rangle$ (|vac $\rangle$ is the empty dot state), one immediately finds

$$
\begin{aligned}
\left\langle 0\left|H_{\mathrm{int}}\right| 0\right\rangle & =\left\langle 1\left|H_{\mathrm{int}}\right| 1\right\rangle \\
& =\sum_{\boldsymbol{k}} D_{\mathrm{e}} \sqrt{\frac{\hbar k}{2 \rho V c_{\mathrm{l}}}} \mathcal{F}_{\mathrm{e}}(\boldsymbol{k})\left(b_{\boldsymbol{k}}+b_{-\boldsymbol{k}}^{\dagger}\right)
\end{aligned}
$$

For the trion state $|2\rangle=a_{\mathrm{e}, \uparrow}^{\dagger} a_{\mathrm{e}, \downarrow}^{\dagger} a_{\mathrm{h}, \uparrow}^{\dagger}|\mathrm{vac}\rangle$, one has

$$
\begin{aligned}
& \left\langle 2\left|H_{\text {int }}\right| 2\right\rangle= \\
& \sum_{\boldsymbol{k}} \sqrt{\frac{\hbar k}{2 \rho V c_{\mathrm{l}}}}\left[2 D_{\mathrm{e}} \mathcal{F}_{\mathrm{e}}(\boldsymbol{k})-D_{\mathrm{h}} \mathcal{F}_{\mathrm{h}}(\boldsymbol{k})\right]\left(b_{\boldsymbol{k}}+b_{-\boldsymbol{k}}^{\dagger}\right) .
\end{aligned}
$$

In order to further simplify the calculations, we neglect the difference between the localization sizes of the electron and hole wave functions. Taking into account the small variation of the electron and hole confinement widths $l_{\mathrm{e}}$ and $l_{\mathrm{h}}$ leads only to inessential quantitative corrections $^{37}$. This leads to the form factors $\mathcal{F}_{\mathrm{e}}(\boldsymbol{k})=$ $\mathcal{F}_{\mathrm{h}}(\boldsymbol{k})=\mathcal{F}(\boldsymbol{k})[$ Eq. (6)] $]$ and to Eq. (2) with $f_{00}=f_{11}=$ $\sqrt{\frac{\hbar k}{2 \rho V c_{1}}} D_{\mathrm{e}} \mathcal{F}(\boldsymbol{k})$ and $f_{22}=\sqrt{\frac{\hbar k}{2 \rho V c_{1}}}\left(2 D_{\mathrm{e}}-D_{\mathrm{h}}\right) \mathcal{F}(\boldsymbol{k})$, and thus to Eq. (4).

With the isotropic acoustic phonon dispersion, we obtain the spectral density of the phonon reservoir $R_{\mathrm{ph}}(\omega)$ [Eq. (20)]:

$$
\begin{aligned}
R_{\mathrm{ph}}(\omega)= & {\left[n_{\mathrm{B}}(\omega)+1\right] \frac{V}{(2 \pi)^{3} \hbar^{2}} \int_{0}^{2 \pi} d \eta \int_{-\pi / 2}^{\pi / 2} d \zeta \cos \zeta } \\
& \times \int d k k^{2}\left|F_{22}(\boldsymbol{k})\right|^{2}\left[\delta\left(\omega-\omega_{\boldsymbol{k}}\right)+\delta\left(\omega+\omega_{\boldsymbol{k}}\right)\right],
\end{aligned}
$$

where the angles $\eta$ and $\zeta$ denote the orientation of the $\boldsymbol{k}$ vector. We can rewrite it in the form

$$
R_{\mathrm{ph}}(\omega)=R_{0}\left[n_{\mathrm{B}}(\omega)+1\right] \omega^{3} g(\omega),
$$

where

$$
R_{0}=\frac{\hbar\left(D_{\mathrm{e}}-D_{\mathrm{h}}\right)^{2}}{8 \pi^{2} \rho c_{1}^{5}}
$$

and the function $g(\omega)$ is defined as

$$
\begin{aligned}
& g(\omega)= \\
& \quad \int_{-\pi / 2}^{\pi / 2} d \zeta \cos \zeta \exp \left[-\frac{l^{2} \omega^{2}}{2 c_{1}^{2}}\left(\cos ^{2} \zeta+\frac{l_{z}^{2}}{l^{2}} \sin ^{2} \zeta\right)\right] .
\end{aligned}
$$

APPENDIX B: LINDBLAD MASTER EQUATION FOR THE TRION RECOMBINATION CHANNEL

In this Appendix, we derive the results for the trion recombination channel in the Lindblad formalism and compare them with those calculated by means of the perturbative method discussed in this paper.

From the interaction Hamiltonian $H_{\mathrm{c}-\mathrm{rad}}$ [Eq. (7)], we derive the Lindblad equation ${ }^{38}$ in the form

$$
\dot{\rho}=\Gamma\left(\sigma_{-} \rho \sigma_{+}-\frac{1}{2} \sigma_{+} \sigma_{-} \rho-\frac{1}{2} \rho \sigma_{+} \sigma_{-}\right)-i\left[H_{\mathrm{ad}}, \rho\right],
$$

where

$$
\begin{aligned}
& \sigma_{+}= \\
& \quad \frac{1}{2}\left[e^{-i \omega_{0} t}(|2\rangle\langle B|+| 2\rangle\langle D|)+e^{-i \omega_{1} t}(|2\rangle\langle B|-| 2\rangle\langle D|)\right]
\end{aligned}
$$

and $H_{\mathrm{ad}}=i \dot{U}_{\mathrm{C}}(t) U_{\mathrm{C}}^{\dagger}(t)$ is the Hamiltonian generating the adiabatic evolution, with $U_{\mathrm{C}}$ given by Eq. (9). This equation is consistent with the perturbative approximation in the sense that the latter is reproduced upon transforming to the interaction picture and performing an expansion in carrier-phonon coupling.

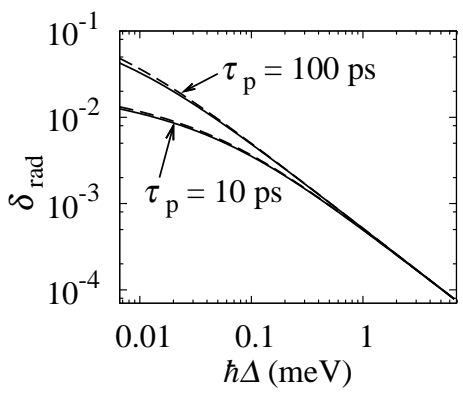

FIG. 11: The radiative error calculated by means of the Lindblad (solid lines) and perturbative (dashed lines) methods for growing detuning.

The results from the Lindblad equation together with those calculated with the perturative theory are plotted in Fig. 11 for the initial state $\left|\psi_{0}\right\rangle=|B\rangle$ for the $\pi / 2$ rotation about the $z$ axis. For small detunings $(\hbar \Delta<0.1 \mathrm{meV})$, where the error is relatively large, the perturbative method yields slightly larger errors, while in the case of larger detunings the results are the same. 
* Electronic address: anna.grodecka@pwr.wroc.pl

1 T. Calarco, A. Datta, P. Fedichev, E. Pazy, and P. Zoller, Phys. Rev. A 68, 12310 (2003).

2 A. Imamoglu, D. D. Awschalom, G. Burkard, D. P. DiVincenzo, D. Loss, M. Sherwin, and A. Small, Phys. Rev. Lett. 83, 4204 (1999).

3 M. Feng, I. D'Amico, P. Zanardi, and F. Rossi, Phys. Rev. A 67, 014306 (2003).

${ }^{4}$ R. Hanson, B. Witkamp, L. M. K. Vandersypen, L. H. Willems van Beveren, J. M. Elzerman, and L. P. Kouwenhoven, Phys. Rev. Lett. 91, 196802 (2003).

${ }^{5}$ E. Pazy, E. Biolatti, T. Calarco, I. D'Amico, P. Zanardi, F. Rossi, and P. Zoller, Europhys. Lett. 62, 175 (2003).

6 C. Emary and L. J. Sham, J. Phys. Cond. Matt. 19, 056203 (2007).

7 S. E. Economou, L. J. Sham, Y. Wu, and D. G. Steel, Phys. Rev. B 74, 205415 (2006).

8 A. Nazir, B. W. Lovett, S. D. Barrett, T. P. Spiller, and G. A. D. Briggs, Phys. Rev. Lett. 93, 150502 (2004).

9 B. W. Lovett, A. Nazir, E. Pazy, S. D. Barrett, T. P. Spiller, and G. A. D. Briggs, Phys. Rev. B 72, 115324 (2005).

10 M. V. G. Dutt, J. Cheng, B. Li, X. Xu, X. Li, P. R. Berman, D. G. Steel, A. S. Bracker, D. Gammon, S. E. Economou, R.-B. Liu, and L. J. Sham, Phys. Rev. Lett. 94, 227403 (2005).

11 A. Greilich, R. Oulton, E. A. Zhukov, I. A. Yugova, D. R. Yakovlev, M. Bayer, A. Shabaev, A. L. Efros, I. A. Merkulov, V. Stavarache, D. Reuter, and A. Wieck, Phys. Rev. Lett. 96, 227401 (2006).

12 M. V. G. Dutt, J. Cheng, Y. Wu, X. Xu, D. G. Steel, A. S. Bracker, D. Gammon, S. E. Economou, R.-B. Liu, and L. J. Sham, Phys. Rev. B 74, 125306 (2006).

13 M. Atatüre, J. Dreiser, A. Badolato, and A. Imamoglu, Nature Phys. 3, 101 (2007).

14 Pochung Chen, C. Piermarocchi, L. J. Sham, D. Gammon, and D. G. Steel, Phys. Rev. B 69, 075320 (2004).

15 F. Troiani, E. Molinari, and U. Hohenester, Phys. Rev. Lett. 90, 206802 (2003).

16 D. Parodi, M. Sassetti, P. Solinas, P. Zanardi, and N. Zanghi, Phys. Rev. A 73, 052304 (2006).

17 K. Roszak, A. Grodecka, P. Machnikowski, and T. Kuhn, Phys. Rev. B 71, 195333 (2005).

18 A. Grodecka, L. Jacak, P. Machnikowski, and K. Roszak, in Quantum Dots: Research Developments, edited by P. A.
Ling (Nova Science Publishers, NY, 2005), p. 47.

19 X. Caillet and C. Simon, Eur. Phys. J. D 42, 341 (2007).

20 M. O. Scully and M. S. Zubairy, Quantum Optics (Cambridge University Press, Cambridge, 1997).

21 V. N. Golovach, A. Khaetskii, and D. Loss, Phys. Rev. Lett. 93016601 (2004).

22 A. Vagov, V. M. Axt, and T. Kuhn, Phys. Rev. B 66, 165312 (2002).

23 L. Jacak, P. Machnikowski, J. Krasnyj, and P. Zoller, Eur. Phys. J. D 22, 319 (2003).

24 P. Machnikowski, V. M. Axt, and T. Kuhn, Phys. Rev. A 75, 052330 (2007).

25 B. Krummheuer, V. M. Axt, and T. Kuhn, Phys. Rev. B 65, 195313 (2002).

26 A. Messiah, Quantum Mechanics (North-Holland, Amsterdam, 1966).

27 M. A. Nielsen and I. L. Chuang, Quantum Computation and Quantum Information (Cambridge University Press, Cambridge, 2000).

28 C. Cohen-Tannoudji, J. Dupont-Roc, and G. Grynberg, Atom-Photon Interactions (Wiley-Interscience, New York, 1998).

${ }^{29}$ R. Alicki, M. Horodecki, P. Horodecki, and R. Horodecki, Phys. Rev. A 65, 062101 (2002).

${ }^{30}$ V. M. Axt, P. Machnikowski, and T. Kuhn, Phys. Rev. B 71, 155305 (2005).

31 A. Krügel, V. M. Axt, T. Kuhn, P. Machnikowski, and A. Vagov, Appl. Phys. B 81, 897 (2005).

32 R. Alicki, M. Horodecki, P. Horodecki, R. Horodecki, L. Jacak, and P. Machnikowski, Phys. Rev. A 70, 010501(R) (2004).

33 P. Machnikowski and L. Jacak, Phys. Rev. B 69, 193302 (2004).

34 J. Förstner, C. Weber, J. Danckwerts, and A. Knorr, Phys. Rev. Lett. 91, 127401 (2003).

35 M. Wenin and W. Potz, Phys. Rev. A 74, 022319 (2006).

36 U. Hohenester and G. Stadler, Phys. Rev. Lett. 92, 196801 (2004).

37 A. Grodecka and P. Machnikowski, Phys. Rev. B 73, 125306 (2006).

38 H.-P. Breuer and F. Petruccione, The Theory of Open Quantum Systems (Oxford University Press, Oxford, 2002). 